See discussions, stats, and author profiles for this publication at: https://www.researchgate.net/publication/243092049

\title{
The basis properties of eigenfunctions in the eigenvalue problem with a spectral parameter in the boundary condition
}

Article in Doklady Mathematics · February 2007

DOI: 10.1134/S1064562407010048

CITATIONS

READS

7

30

2 authors:

Af Nazim B. Kerimov

Mersin University,Mersin, Turkey

48 PUBLICATIONS 516 CITATIONS

SEE PROFILE

Ziyatkhan S. Aliev

Baku State University

59 PUBLICATIONS 323 CITATIONS

SEE PROFILE

Some of the authors of this publication are also working on these related projects:

Sturm-Liouville View project 


\title{
The Basis Properties of Eigenfunctions in the Eigenvalue Problem with a Spectral Parameter in the Boundary Condition
}

\author{
N. B. Kerimov ${ }^{a}$ and Z. S. Aliev ${ }^{b}$ \\ Presented by Academician E.I. Moiseev July 21, 2006
}

Received January 13, 2006

DOI: $10.1134 / \mathrm{S} 1064562407010048$

Consider the eigenvalue problem

$$
\begin{gathered}
y^{\mathrm{IV}}-\left(q(x) y^{\prime}\right)^{\prime}=\lambda y, \quad 0<x<l, \\
y^{\prime}(0) \cos \alpha-y^{\prime \prime}(0) \sin \alpha=0, \\
y(0) \cos \beta+T y(0) \sin \beta=0, \\
y(l) \cos \gamma+y^{\prime \prime}(l) \sin \gamma=0, \\
(a \lambda+b) y(l)-(c \lambda+d) T y(l)=0,
\end{gathered}
$$

where $\lambda$ is the spectral parameter; $T y \equiv y^{\prime \prime \prime}-q y^{\prime} ; q(x)$ is a absolutely continuous positive function on the interval $[0, l]$; and $\alpha, \beta, \gamma, a, b, c$, and $d$ are real constants such that $0 \leq \alpha, \beta, \gamma \leq \frac{\pi}{2}$.

In what follows, we assume that

$$
\sigma=b c-a d>0 \text {. }
$$

Boundary value problems for second- and fourthorder ordinary differential operators with a spectral parameter in the boundary conditions have been extensively studied (see, e.g., [1-9]). In [3-5], such problems were associated with particular physical processes.

The basis properties of the system of eigenfunctions in the Sturm-Liouville problem with a spectral parameter in the boundary conditions were studied in various function spaces in [7-9]. The existence of eigenvalues, estimate for eigenvalues and eigenfunctions, and expansion theorems for fourth-order operators with a spectral parameter in the boundary condition were considered in $[1,6]$.

\footnotetext{
${ }^{a}$ Institute of Mathematics and Mechanics, National Academy of Sciences of Azerbaijan, Baku, Azerbaijan e-mail:nazimkerimov@yahoo.com

${ }^{b}$ Baku State University, Baku, Azerbaijan e-mail: z_aliyev@mail.ru
}

This paper deals with the basis properties in $L_{p}(0, l)$ $(1<p<\infty)$ of the system of eigenfunctions of boundary value problem (1), (2).

\section{ASYMPTOTIC FORMULAS \\ FOR EIGENVALUES AND EIGENFUNCTIONS \\ OF BOUNDARY VALUE PROBLEM (1), (2)}

We introduce the boundary condition

$$
y(l) \cos \delta-T y(l) \sin \delta=0, \quad \delta \in[0, \pi / 2] .
$$

and, along with problem (1), (2), consider boundary value problem (1), (2a)-(2c), (2d').

Theorem 1 [10]. The eigenvalues of boundary value problem (1), (2a)-(2c), (2d') are simple and form an infinitely increasing sequence $0 \leq \mu_{1}(\delta)<\mu_{2}(\delta)<\ldots<$ $\mu_{n}(\delta)<\ldots$ Moreover, the eigenfunction $V_{n}^{(\delta)}(x)$ corresponding to the eigenvalue $\mu_{n}(\delta)$ has exactly $n-1$ simple zeros in the interval $(0, l)$.

The numbers $\tau, \rho, \tau_{n}, \rho_{n}(n \in \mathbb{N})$ and the function $z(x, t), x \in[0, l], t \in \mathbb{R}$ are defined as

$$
\begin{gathered}
=\left\{\begin{array}{l}
\frac{3(1+\operatorname{sgn} \beta+\operatorname{sgn} \delta)}{4}-1, \text { if } \operatorname{sgn} \alpha+\operatorname{sgn} \gamma=1, \\
\frac{5+2 \operatorname{sgn} \alpha}{4}+\left((-1)^{\operatorname{sgn} \alpha+\operatorname{sgn} \beta}+(-1)^{\operatorname{sgn} \alpha+\operatorname{sgn} \delta}\right) \\
\times \frac{6 \operatorname{sgn} \alpha-3}{8}-1, \text { if } \operatorname{sgn} \alpha+\operatorname{sgn} \gamma \neq 1,
\end{array}\right. \\
\rho=\left\{\begin{array}{l}
\frac{3(1+\operatorname{sgn} \beta+\operatorname{sgn}|c|)}{4}, \text { if } \operatorname{sgn} \alpha+\operatorname{sgn} \gamma=1, \\
\frac{5+2 \operatorname{sgn} \alpha}{4}+\left((-1)^{\operatorname{sgn} \alpha+\operatorname{sgn} \beta}+(-1)^{\operatorname{sgn} \alpha+\operatorname{sgn}|c|}\right) \\
\times \frac{6 \operatorname{sgn} \alpha-3}{8}, \quad \text { if } \operatorname{sgn} \alpha+\operatorname{sgn} \gamma \neq 1,
\end{array}\right.
\end{gathered}
$$




$$
\begin{gathered}
\tau_{n}=(n-\tau) \frac{\pi}{l}, \quad \rho_{n}=(n-\rho) \frac{\pi}{l}, \\
z(x, t)=\left\{\begin{array}{l}
\sin \left(t x+\frac{\pi}{2} \operatorname{sgn} \beta\right) \\
-\cos \left(t l+\frac{\pi}{2}(\operatorname{sgn} \beta+\operatorname{sgn} \gamma)\right) \exp (-t(l-x)), \\
\text { if } \operatorname{sgn} \alpha+\operatorname{sgn} \beta=1, \\
\sin t x-\cos t x+(-1)^{\operatorname{sgn} \alpha} \exp (-t x) \\
+\sqrt{2} \times(-1)^{1-\operatorname{sgn} \gamma} \sin \left(t l+\frac{\pi}{4} \times(-1)^{\operatorname{sgn} \gamma}\right) \\
\times \exp (-t(l-x)), \text { if } \operatorname{sgn} \alpha+\operatorname{sgn} \beta \neq 1 .
\end{array}\right.
\end{gathered}
$$

Theorem 2. It holds that

$$
\begin{gathered}
\sqrt[4]{\mu_{n}(\delta)}=\tau_{n}+O\left(\frac{1}{n}\right), \\
V_{n}^{(\delta)}(x)=z\left(x, \tau_{n}\right)+O\left(\frac{1}{n}\right) .
\end{gathered}
$$

The proof of Theorem 2 is based on Theorem 1 and formulas (45.a) and (45.b) in [11].

Theorem 3. The spectrum of boundary value problem (1), (2) consists of an infinite sequence of simple eigenvalues $\lambda_{1}<\lambda_{2}<\ldots<\lambda_{n}<\ldots$ such that $\lambda_{n}>0$ for $n \geq 3$. For the eigenvalues $\lambda_{n}$ and the corresponding eigenfunctions $y_{n}(x)$, we have the asymptotic formulas

$$
\begin{gathered}
\sqrt[4]{\lambda_{n}}=\rho_{n}+O\left(\frac{1}{n}\right), \\
y_{n}(x)=z\left(x, \rho_{n}\right)+O\left(\frac{1}{n}\right) .
\end{gathered}
$$

The existence of eigenvalues of boundary value problem (1), (2) follows from Theorem 1 and the lemma below.

Lemma 1. Let $y(x, \lambda)$ be a nontrivial solution to problem (1), (2a)-(2c).

Then, in each interval $\left(\mu_{n-1}(0), \mu_{n}(0)\right)$, where $n \in \mathbb{N}$ and $\mu_{0}(0)=-\infty$, the function $\frac{T y(l, \lambda)}{y(l, \lambda)}$ is continuous and strictly increasing. Moreover,

$$
\lim _{\lambda \rightarrow-\infty} \frac{T y(l, \lambda)}{y(l, \lambda)}=-\infty
$$

The proofs of asymptotic formulas (5) and (6) are based on Theorem 1 in [11], Theorem 2, and the following lemma.

Lemma 2. For sufficiently large $n \in \mathbb{N}$,

$$
\begin{gathered}
\mu_{n-2}(0)<\mu_{n-1}\left(\frac{\pi}{2}\right)<\lambda_{n}<\mu_{n-1}(0), \\
\text { if } c \neq 0 \quad u \frac{a}{c} \geq 0, \\
\mu_{n-2}(0)<\lambda_{n}<\mu_{n-1}\left(\frac{\pi}{2}\right)<\mu_{n-1}(0), \\
\text { if } c \neq 0 u \frac{a}{c}<0, \\
\mu_{n-1}(0)<\lambda_{n}<\mu_{n}\left(\frac{\pi}{2}\right)<\mu_{n}(0), \text { if } \quad c=0 .
\end{gathered}
$$

2. BASIS PROPERTY IN $L_{p}(0, l)(1<p<\infty)$ OF THE SYSTEM OF EIGENFUNCTIONS OF BOUNDARY VALUE PROBLEM (1), (2)

Theorem 4. Let $r$ be an arbitrary fixed positive integer.

Then the system $\left\{y_{n}(x)\right\}(n=1,2, \ldots ; n \neq r)$ is minimal in $L_{p}(0, l)(1<p<\infty)$.

Proof sketch of Theorem 4. It is sufficient to prove the existence of a system $\left\{u_{n}(x)\right\}(n=1,2, \ldots ; n \neq r)$ that is biorthogonal adjoint to $\left\{y_{n}(x)\right\}(n=1,2, \ldots ; n \neq r)$.

Let $c=0$. The biorthogonal adjoint system is given by the relation

$$
u_{n}(x)=\left(y_{n}(x)-\frac{y_{n}(l)}{y_{r}(l)} y_{r}(x)\right) /\left(\left\|y_{n}\right\|_{2}^{2}-\frac{a}{d} y_{n}^{2}(l)\right),
$$

where $\|\cdot\|_{p}$ denotes the norm in $L_{p}(0, l)$.

Let $c \neq 0$. The number $N$ is determined by the inequality $\mu_{N-1}(0)<-\frac{d}{c} \leq \mu_{N}(0)$. When $\lambda_{N+1} \neq-\frac{d}{c}$, the biorthogonal adjoint system is

$$
\begin{gathered}
u_{n}(x)=\left(y_{n}(x)-\frac{\left(c \lambda_{r}+d\right) y_{n}(l)}{\left(c \lambda_{n}+d\right) y_{r}(l)} y_{r}(x)\right) /\left(\left\|y_{n}\right\|_{2}^{2}\right. \\
\left.+\frac{\sigma y_{n}^{2}(l)}{\left(c \lambda_{n}+d\right)^{2}}\right) .
\end{gathered}
$$

When $\lambda_{N+1}=-\frac{d}{c}$, the biorthogonal adjoint system is given by

$$
\begin{gathered}
u_{n}(x) \\
=\left(y_{n}(x)-\frac{\sigma y_{n}(l)}{c\left(c \lambda_{n}+d\right) T y_{N+1}(l)} y_{N+1}(x)\right) /\left(\left\|y_{n}\right\|_{2}^{2}\right. \\
\left.+\frac{\sigma y_{n}^{2}(l)}{\left(c \lambda_{n}+d\right)^{2}}\right)
\end{gathered}
$$


for $r=N+1$, by (8) for $r \neq N+1$ and $n \neq N+1$, and by

$$
u_{n}(x)=\frac{y_{N+1}(x)-\frac{c\left(c \lambda_{r}+d\right) T y_{N+1}(l)}{\sigma y_{r}(l)} y_{r}(x)}{\left\|y_{N+1}\right\|^{2}+\frac{c^{2}\left(T y_{N+1}(l)\right)^{2}}{\sigma}}
$$

for $r \neq N+1$ and $n=N+1$.

Theorem 4 is proved.

Taking into account (5) and (6), we find from (7)(9) the asymptotic formula

$$
u_{n}(x)=l^{-1} y_{n}(x)+O\left(\frac{1}{n}\right) .
$$

Below is the main result of this paper.

Theorem 5. Let $r$ be an arbitrary fixed positive integer.

Then the system $\left\{y_{n}(x)\right\}(n=1,2, \ldots ; n \neq r)$ forms a basis in $L_{p}(0, l)(1<p<\infty)$, and this basis is unconditional for $p=2$.

Proof sketch of Theorem 5. The boundary conditions $(2 \mathrm{a})-(2 \mathrm{c}),\left(2 \mathrm{~d}^{\prime}\right)$ are strongly regular (see [11]). Then, by Theorem 5.1 in [12], the system of eigenfunctions $\left\{v_{n}^{(\delta)}(x)\right\}_{n=1}^{\infty}$ of problem (1), (2a)-(2c), (2d') forms a basis in $L_{p}(0, l)(1<p<\infty)$ and this basis is unconditional for $p=2$.

Let $c=0$. We compare the system $\left\{y_{n}(x)\right\}(n=1,2$, $\ldots ; n \neq r)$ with $\left\{v_{n}^{(0)}(x)\right\}_{n=1}^{\infty}$. By virtue of (4) and (6), for sufficiently large $n$, it holds that

$$
\left\|y_{n+1}(x)-V_{n}^{(0)}(x)\right\|_{2} \leq \text { const } \cdot n^{-1},
$$

which implies the convergence of the series

$$
\sum_{n=1}^{r-1}\left\|y_{n}(x)-v_{n}^{(0)}(x)\right\|_{2}^{2}+\sum_{n=r}^{\infty}\left\|y_{n+1}(x)-v_{n}^{(0)}(x)\right\|_{2}^{2}
$$

(for $r=1$, the first sum is absent). Consequently, $\left\{y_{n}(x)\right\}$ $(n=1,2, \ldots ; n \neq r)$ is quadratically close to the system $\left\{v_{n}^{(0)}(x)\right\}_{n=1}^{\infty}$. By Theorem 4, the system $\left\{y_{n}(x)\right\}(n=$ $1,2, \ldots ; n \neq r)$ is minimal in $L_{p}(0, l)(1<p<\infty)$. Then, by Theorem 9.9.8 in [13], the system $\left\{y_{n}(x)\right\}(n=1$, $2, \ldots ; n \neq r)$ is an unconditional basis in $L_{2}(0, l)$.

The case $c \neq 0$ is considered in a similar manner, with the system $\left\{y_{n}(x)\right\}(n=1,2, \ldots ; n \neq r)$ compared with $\left\{v_{n}^{(\pi / 2)}(x)\right\}_{n=1}^{\infty}$.
Let $\tilde{V}_{n}^{(\delta)}(x)=v_{n}^{(\delta)}(x)\left\|v_{n}^{(\delta)}(x)\right\|_{2}^{-1}(n=1,2, \ldots)$. Since boundary value problem (1), (2a)-(2c), (2d") is selfadjoint, it follows from (4) that the systems $\left\{\tilde{V}_{n}^{(0)}(x)\right\}_{n=1}^{\infty}=1$ and $\left\{v_{n}^{(\pi / 2)}(x)\right\}_{n=1}^{\infty}$ are uniformly bounded orthonormal bases in $L_{2}(0, l)$.

By using (4), (6), and (10), it is easy to see that

$$
\begin{aligned}
& y_{n}(x)=l^{1 / 2} \tilde{V}_{n}(x)+O\left(\frac{1}{n}\right), \\
& u_{n}(x)=l^{-1 / 2} \tilde{V}_{n}(x)+O\left(\frac{1}{n}\right)
\end{aligned}
$$

for $n \in \mathbb{N}$ and $n \neq r$. Here,

$$
\tilde{V}_{n}(x)=\left\{\begin{array}{l}
\tilde{V}_{n}^{(0)}(x) \text { for } c=0 \text { and } 1 \leq n \leq r-1 ; \\
\tilde{V}_{n-1}^{(0)}(x) \text { for } c=0 \text { and } n \geq r-1 ; \\
\tilde{V}_{n}^{(\pi / 2)}(x) \text { for } c \neq 0 \text { and } 1 \leq n \leq r-1 ; \\
\tilde{V}_{n-1}^{(\pi / 2)}(x) \text { for } c \neq 0 \text { and } n \geq r+1 .
\end{array}\right.
$$

We now fix $p \in(1,2)$. Since $\left\{y_{n}(x)\right\}(n=1,2, \ldots$; $n \neq r)$ is a basis in $L_{2}(0, l)$, this system is complete in $L_{p}(0, l)$. Using (11) and Theorem 2.3 in [14], we can prove the estimate

$$
\left\|\sum_{n=1, n \neq r}^{k}\left(f, u_{n}\right) y_{n}\right\|_{p} \leq M_{p}\|f\|_{p}, \quad k=1,2, \ldots
$$

for an arbitrary function $f(x)$ in $L_{p}(0, l)$, where $M_{p}$ is a positive constant. By Theorem 6 in [15], the system $\left\{y_{n}(x)\right\}(n=1,2, \ldots ; n \neq r)$ is a basis in $L_{p}(0, l)$.

Let $2<p<+\infty$. Following the above line of reasoning, we prove the basis property of $\left\{u_{n}(x)\right\}(n=1,2, \ldots$; $n \neq r)$ in $L_{q}(0, l)\left(q=\frac{p}{p-1}\right)$, which is equivalent to the basis property of $\left\{y_{n}(x)\right\}(n=1,2, \ldots ; n \neq r)$ in $L_{p}(0, l)$ (see [15]). Theorem 5 is proved.

\section{REFERENCES}

1. W. Sternberg, Math. Z. 3, 191-208 (1919).

2. C. T. Fulton, Math. Z. 133, 301-312 (1973).

3. G. H. Handleman and J. B. Keller, Proceedings of 4th US National Congress of Applied Mechanics (Am. Soc. Mech. Eng., New York, 1962), pp. 195-202.

4. H. J. Ahn, Q. Appl. Math. 39 (1), 109-117 (1981).

5. M. Roseau, Vibrations in Mechanical Systems (SpringerVerlag, Berlin, 1984).

6. S. V. Meleshko and Yu. V. Pokornyi, Differ. Uravn. 23, 1466-1467 (1987). 
7. N. Yu. Kapustin and E. I. Moiseev, Differ. Equations 36, 1182-1188 (2000) [Differ. Uravn. 36, 1069-1074 (2000)].

8. N. Yu. Kapustin and E. I. Moiseev, Differ. Equations 36, 1498-1501 (2000) [Differ. Uravn. 36, 1357-1360 (2000)].

9. E. B. Kerimov and V. S. Mirzoev, Sib. Mat. Zh. 44, 1041-1045 (2003).

10. D. O. Banks and G. J. Kurowski, J. Differ. Equations 24, 57-74 (1977).

11. M. A. Naimark, Linear Differential Operators (Ungar, New York, 1967; Nauka, Moscow, 1969).
12. H. E. Benzinger, Trans. Am. Math. Soc. 174, 333-344 (1972).

13. S. Kaczmarz and H. Steinhaus, Theorie der Orthogonalreihen (Chelsea, New York, 1951; Fizmatgiz, Moscow, 1958).

14. A. Zygmund, Trigonometric Series (Cambridge Univ. Press, Cambridge, 1960; Mir, Moscow, 1965), Vol. 2.

15. B. S. Kashin and A. A. Saakyan, Orthogonal Series (Nauka, Moscow, 1984; Am. Math. Soc., Providence, R.I., 1989). 